\title{
Eschenmoser-Claisen Rearrangement of Baylis-Hillman Adducts ${ }^{\dagger}$
}

\author{
Jeong Mi Kim. Sung Hwan Kim, and Jae Nyoung Kim" \\ Department of Chemistry and Institute of Basic Science, Chonnam National University, Gwangit 500-757, Korea \\ "E-mail: kimin@ichonnam.ac.kr \\ Received May 16, 2007
}

Key Words : Eschenmoser-Claisen rearrangement, Baylis-Hillman adducts, DMA-DMA

Johnson-Claisen rearangement of the Baylis-Hillman adducts has been reported by Basavaiah and co-workers to produce alkyl alk-4-enoate derivatives stereoselectively. ${ }^{\text {la }}$ Das and co-workers examined the Johnson-Claisen rearrangement of Baylis-Hillman adducts with triethyl orthoacetate and reported the applicability of $\mathrm{I}_{2} / \mathrm{SiO}_{2}$ or $\mathrm{NaHSO}_{4} / \mathrm{SiO}_{2}$ systems. ${ }^{1 t . c}$ We also examined the reaction of aza-BaylisHillman adducts ${ }^{2 \mathrm{a}}$ and Baylis-Hillman adducts ${ }^{2 \mathrm{~b}}$ with $N . N$ dimethylformamide dimethylacetal (DMF-DMA), and we obtained cinnamyl amine derivatives in both cases. ${ }^{2}$

However, literature survey revealed that EschenmoserClaisen rearrangement ${ }^{3}$ of Baylis-Hillman adducts has not been reported. ${ }^{2 b}$ Thus, we decided to examine the Eschenmoser-Claisen rearrangement of Baylis-Hillman adducts (1a-c, $\mathbf{I g}$, and $\mathbf{I h}$ ) and cinnamyl alcohol derivatives (1d-1) with $N, N$-dimethylacetamide dimethylacetal (DMA-DMA) and report herein the results (Scheme 1 shows the synthesis of $\mathbf{2 a}$ and $\mathbf{2} \mathbf{l}$ as representative examples)

Required starting materials $1 \mathbf{a}-\mathbf{h}$ were synthesized accord- ing to the reported methods. ${ }^{1.2 .4}$ The reaction conditions including the amounts of DMA-DMA, solvent, and temperature were examined and we found that optimum yields were obtained with 2.0 equiv of DMA-DMA in toluene at around $80-90^{\circ} \mathrm{C}$. The results are summarized in Table 1 and the reaction mechanism is depicted in Scheme 1. For the secondary alcohol derivatives 1 a-c, some ester compounds (3a: $5 \%$ in entry 1 and $3 \mathbf{b}$ : $4 \%$ in entry 2) were formed together (due to the competitive formation of intermediate III) and the yields of desired products $2 \mathbf{a}-\mathbf{c}$ were moderate $(69-71 \%)$. The products $2 \mathrm{a}$ and $2 \mathrm{~b}$ were isolated as $E / Z$ mixtures ( $c a .9: 1$ ) as in the Johnson-Claisen rearrangement of the Baylis-Hillman adducts.' The product $2 c$ was obtained as a pure $Z$ isomer, however, 2-dimethylaminomethyl-3phenylacrylonitrile $(4, Z \text {-form })^{2 b}$ was isolated together $(13 \%)$. The primary alcohol derivatives $1 \mathbf{d}-\mathbf{f}$ were cleanly converted into $\mathbf{2 d - f}$ in moderate to good yields (75-84\%) and we could not find the conesponding ester derivatives in these cases. The Baylis-Hillman adducts of 2-cyclohexen-1-

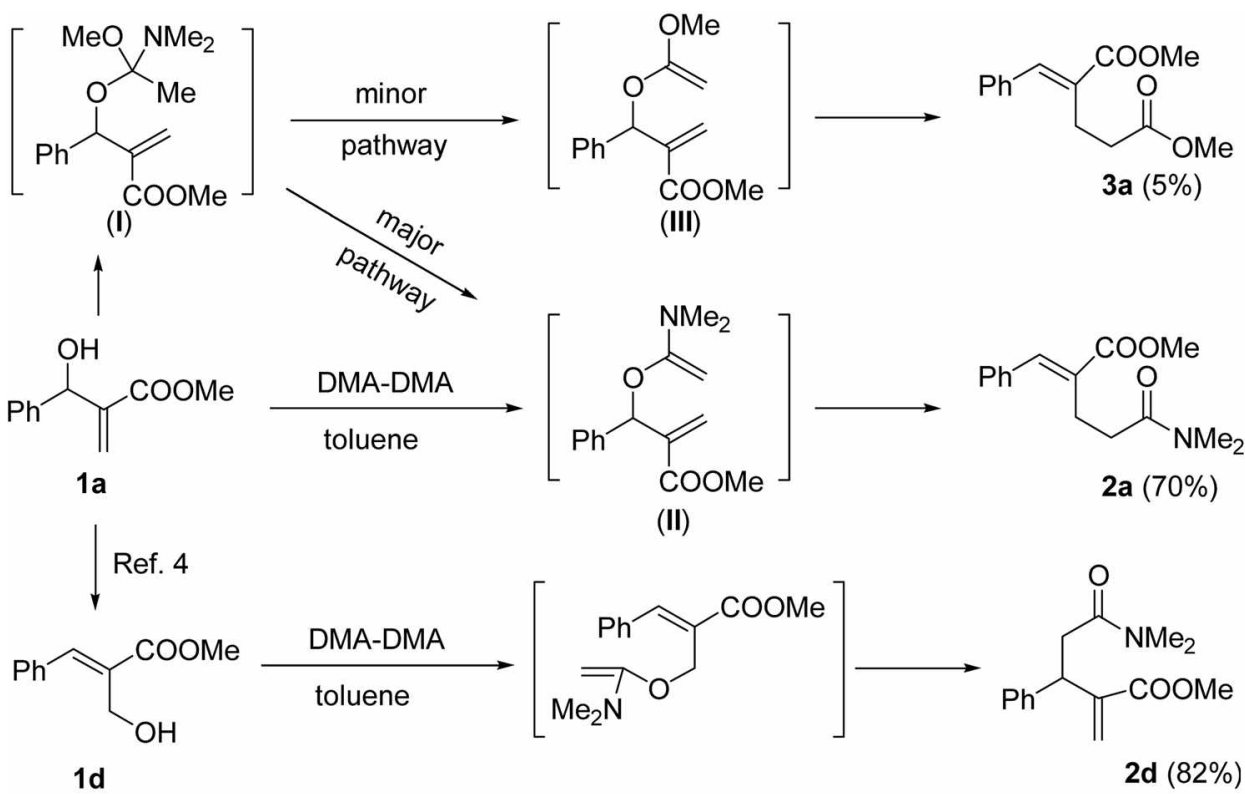

Scheme 1

\footnotetext{
${ }^{7}$ This paper is dedicated to Professor Sang Chul Shim on the occasion of his honorable retirement.
} 
Table 1. Eschenunoser-Claisen rearrangement of Baylis-Hillınan adducts

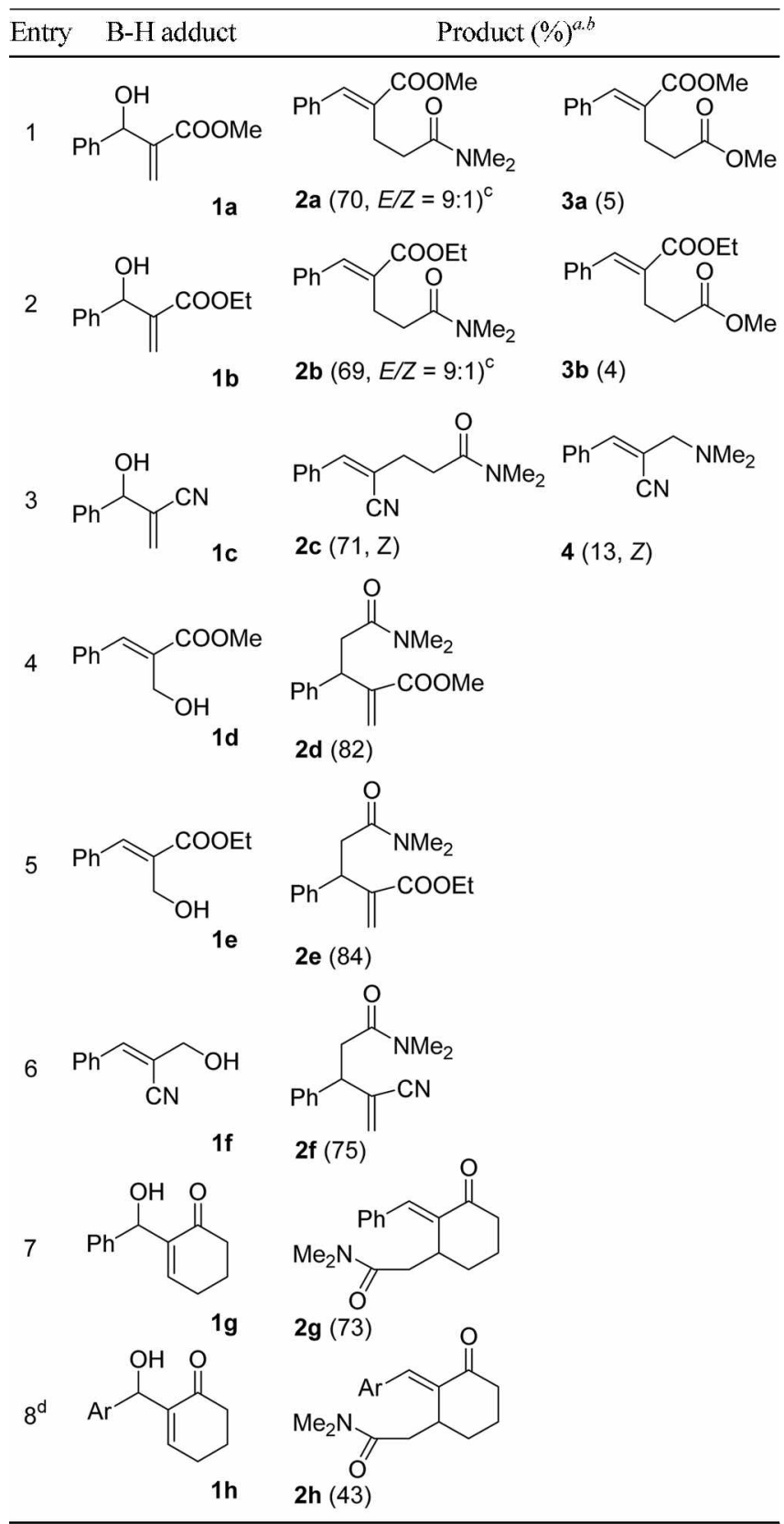

Isolated yields. ${ }^{b}$ The reaction was carried out in toluene with DMADMA (2.0 equiv) at $80-90{ }^{\circ} \mathrm{C}$ for $2 \mathrm{~h}$. ${ }^{\circ}$ The ratio of $\mathrm{E} / \mathrm{Z}$ was determined based on 'II NMR. Ar is 4nitrophenyl.

one, $1 \mathrm{~g}$ and $1 \mathrm{~h}$, were also converted into $2 \mathrm{~g}$ and $2 \mathrm{~h}$, respectively, in moderate yields.

In summary, we disclosed the Eschenmoser-Claisen rearrangement of Baylis-Hillman adducts ${ }^{5}$ and cinnamyl alcohol derivatives with $N, N$-dimethylacetamide-dimethylacetal (DMA-DMA) in toluene to produce 2-benzylidene 1,5-dicarbonyl compounds and 2-methylene 1,5-dicarbonyl derivatives in moderate yields.

\section{Experimental Section}

Typical procedure for the synthesis of $2 a$ and the spectroscopic data of $\mathbf{2 a -}-\mathrm{h}_{3}, \mathbf{3}, \mathbf{3}, \mathrm{b}$, and $\mathbf{4}$ are as follows. A mixture of 1a (192 mg, $1.0 \mathrm{mmol})$ and DMA-DMA (266 $\mathrm{mg}, 2.0 \mathrm{mmol})$ in toluene $(3 \mathrm{~mL})$ was stirred at $80-90^{\circ} \mathrm{C}$ for $2 \mathrm{~h}$. After removal of solvent desired product was separated by column chromatographic purification process (hexanes/ EtOAc, 3:5) as pale yellow oil, $183 \mathrm{mg}(70 \%)$.

Compound 2a: $70 \%(E / Z=9: 1)$; pale yellow oil; IR (film) $2950,1708,1654,1648,1250 \mathrm{~cm}^{-1}$; 'H NMR ( $\mathrm{CDCl}_{3}, 300$ MHz) $\delta 2.53-2.58(\mathrm{~m}, 2 \mathrm{H}), 2.86-2.90(\mathrm{~m}, 2 \mathrm{H}), 2.94(\mathrm{~s}, 3 \mathrm{H})$, $3.00(\mathrm{~s}, 3 \mathrm{H}), 3.83(\mathrm{~s}, 3 \mathrm{H}), 7.25-7.40(\mathrm{~m}, 5 \mathrm{H}), 7.73(\mathrm{~s}, 1 \mathrm{H})$; ${ }^{13} \mathrm{C}$ NMR (CDCl, $\left.75 \mathrm{MHz}\right) \delta 23.52,32.77,35.34,37.16$, $52.05,128.65,128.66,129.29,131.61,135.20,140.24$, $168.62,171.97$.

Compound $2 \mathrm{~b}:{ }^{2 b} 69 \%(E / Z=9: 1)$; pale yellow oil; IR (film) $2935,1704,1650 \mathrm{~cm}^{-1}$; ${ }^{1} \mathrm{H}$ NMR $\left(\mathrm{CDCl}_{3}, 300 \mathrm{MHz}\right)$ $\delta 1.35(\mathrm{t}, J=7.2 \mathrm{~Hz}, 3 \mathrm{H}), 2.53-2.60(\mathrm{~m}, 2 \mathrm{H}), 2.73-2.76(\mathrm{~m}$, $2 \mathrm{H}), 2.94(\mathrm{~s}, 3 \mathrm{H}), 3.00(\mathrm{~s}, 3 \mathrm{H}), 4.28(\mathrm{q}, J=7.2 \mathrm{~Hz}, 2 \mathrm{H})$, $7.30-7.42(\mathrm{~m}, 5 \mathrm{H}), 7.73(\mathrm{~s}, 1 \mathrm{H}) ;{ }^{13} \mathrm{C} \mathrm{NMR}\left(\mathrm{CDCl}_{3}, 75 \mathrm{MHz}\right)$ $\delta 14.29,23.51,32.78,35.29,37.14,60.84,128.55,128.61$, $129.25,130.87,135.25,139.92,168.09,172.00$.

Compound 2c: $71 \%(Z)$; yellow solid, mp $47-49^{\circ} \mathrm{C}$; IR (film) $2919,2203,1650,1644 \mathrm{~cm}^{-1}$; $14 \mathrm{NMR}\left(\mathrm{CDCl}_{3}, 300\right.$ MHz) $\delta 2.66(\mathrm{t}, J=6.9 \mathrm{~Hz}, 2 \mathrm{H}), 2.78(\mathrm{t}, J=6.9 \mathrm{~Hz}, 2 \mathrm{H})$, $2.96(\mathrm{~s}, 3 \mathrm{H}), 3.04(\mathrm{~s}, 3 \mathrm{H}), 7.08(\mathrm{~s}, 1 \mathrm{H}), 7.35-7.44(\mathrm{~m}, 3 \mathrm{H})$, $7.70-7.74(\mathrm{~m}, 2 \mathrm{H}) ;{ }^{13} \mathrm{C}$ NMR $\left(\mathrm{CDCl}_{3}, 75 \mathrm{MHz}\right) \delta 31.45$, $31.64,35.49,37.11,109.82,118.64,128.61,128.76,130.03$, $133.59,144.71,170.63$.

Compound 2d: $82 \%$; white solid, mp $73-74^{\circ} \mathrm{C}$; IR (film) 2945, 1720,1654, I648, $1642 \mathrm{~cm}^{-1}$; 'H NMR (CDCl 300 MHz) $\delta 2.77-2.96(\mathrm{~m}, 2 \mathrm{H}), 2.88(\mathrm{~s}, 3 \mathrm{H}), 2.90(\mathrm{~s}, 3 \mathrm{H}), 3.66$ $(\mathrm{s}, 3 \mathrm{H}), 4.53(\mathrm{t}, J=7.5 \mathrm{~Hz}, 1 \mathrm{H}), 5.63(\mathrm{~s}, 1 \mathrm{H}), 6.32(\mathrm{~s}, 1 \mathrm{H})$, 7.16-7.30 (m, 5H); ${ }^{13} \mathrm{C} \mathrm{NMR}\left(\mathrm{CDCl}_{3}, 75 \mathrm{MHz}\right) \delta 35.46$, $37.15,38.06,43.06,51.83,124.33,126.63,127.81,128.41$, $142.03,142.89,166.98,170.67$.

Compound 2e: $84 \%$; white solid, mp $63-64^{\circ} \mathrm{C}$; IR (film) 2976, 2925, 1716, I651, $1649 \mathrm{~cm}^{-1}$; $1 \mathrm{H}$ NMR ( $\mathrm{CDCl}_{3}, 300$ MHz) $\delta 1.18(\mathrm{t}, J=7.2 \mathrm{~Hz}, 3 \mathrm{H}), 2.77-2.95(\mathrm{~m}, 2 \mathrm{H}), 2.88(\mathrm{~s}$, $3 \mathrm{H}), 2.89(\mathrm{~s}, 3 \mathrm{H}), 4.04-4.15(\mathrm{~m}, 2 \mathrm{H}), 4.52(\mathrm{t}, J=7.5 \mathrm{~Hz}$, $1 \mathrm{H}), 5.61(\mathrm{~s}, 1 \mathrm{H}), 6.32(\mathrm{~s}, 1 \mathrm{H}), 7.16-7.30(\mathrm{~m}, 5 \mathrm{H}) ;{ }^{13} \mathrm{C} \mathrm{NMR}$ $\left(\mathrm{CDCl}_{3}, 75 \mathrm{MHz}\right) \delta 14.00,35.47,37.17,38.04,43.14,60.69$, $123.97,126.60,127.86,128.36,142.16,143.17,166.53$, 170.74 .

Compound 2f: $75 \%$; pale yellow solid, $\mathrm{mp} 71-72{ }^{\circ} \mathrm{C}$; IR (film) 2924, 2211, 1647 $\mathrm{cm}^{-1}$; 'HNMR (CDCl $\left.3,300 \mathrm{MHz}\right) \delta$ 2.77-3.08 (m, 2H), $2.93(\mathrm{~s}, 3 \mathrm{H}), 3.02(\mathrm{~s}, 3 \mathrm{H}), 4.24-4.29(\mathrm{~m}$, $1 \mathrm{H}), 5.60(\mathrm{~s}, 1 \mathrm{H}), 5.93(\mathrm{~s}, 1 \mathrm{H}), 7.25-7.38(\mathrm{~m}, 5 \mathrm{H}) ;{ }^{13} \mathrm{C} \mathrm{NMR}$ $\left(\mathrm{CDCl}_{3}, 75 \mathrm{MHz}\right) \delta 35.59,36.80,37.20,46.05,117.92$, $126.10,127.43,127.60,128.97,131.29,140.16,169.54$.

Compound 2g: 73\%; pale yellow oil; IR (film) 2936, $1681,1643 \mathrm{~cm}^{-1}$; ${ }^{7} \mathrm{H}$ NMR (CDCl $\left.3,300 \mathrm{MHz}\right) \delta 1.83-2.04$ $(\mathrm{m}, 4 \mathrm{H}), 2.25-2.50(\mathrm{~m}, 2 \mathrm{H}), 2.58-2.66(\mathrm{~m}, 2 \mathrm{H}), 2.88(\mathrm{~s}, 3 \mathrm{H})$, $2.92(\mathrm{~s}, 3 \mathrm{H}), 4.01-4.08(\mathrm{~m}, \mathrm{lH}), 7.27-7.48(\mathrm{~m}, 6 \mathrm{H}) ;{ }^{13} \mathrm{C}$ NMR $\left(\mathrm{CDCl}_{3}, 75 \mathrm{MHz}\right) \delta 19.21,27.49,33.34,35.38,35.95$, $37.11,40.51,128.63,128.78,129.84,134.94,135.02$, $141.34,170.54,202.97$.

Compound 2h: 43\%; pale yellow oil; IR (film) 2930, $1689,1644 \mathrm{~cm}^{-1}$; 1 N NMR (CDCl $\left.3,300 \mathrm{MHz}\right) \delta 1.88-2.04$ 
$(\mathrm{m}, 4 \mathrm{H}), 2.23-2.30(\mathrm{~m}, 1 \mathrm{H}), 2.41-2.50(\mathrm{~m}, 1 \mathrm{H}), 2.60-2.72$ $(\mathrm{m}, 2 \mathrm{H}), 2.93(\mathrm{~s}, 6 \mathrm{H}), 4.00-4.04(\mathrm{~m}, 1 \mathrm{H}), 7.24(\mathrm{~s}, 1 \mathrm{H}), 7.60$ (d, $J=8.4 \mathrm{~Hz}, 2 \mathrm{H}), 8.25$ (d, $J=8.4 \mathrm{~Hz}, 2 \mathrm{H}$ ); ${ }^{13} \mathrm{C}$ NMR $\left(\mathrm{CDCl}_{3}, 75 \mathrm{MHz}\right) \delta 19.39,27.76,33.76,35.58,35.74,37.26$, $40.79,123.88,130.43,131.75,141.59,144.94,147.29$, $170.06,202.78$.

Compound 3a: 5\%; colorless oil; 'H NMR $\left(\mathrm{CDCl}_{3}, 300\right.$ MHz) $\delta 2.54-2.60(\mathrm{~m}, 2 \mathrm{H}), 2.85-2.91(\mathrm{~m}, 2 \mathrm{H}), 3.65(\mathrm{~s}, 3 \mathrm{H})$, $3.83(\mathrm{~s}, 3 \mathrm{H}), 7.28-7.43(\mathrm{~m}, 5 \mathrm{H}), 7.74(\mathrm{~s}, 1 \mathrm{H})$.

Compound 3b: 4\%; colorless oil; ${ }^{1} \mathrm{H}$ NMR $\left(\mathrm{CDCl}_{3}, 300\right.$ $\mathrm{MHz}) \delta 1.35(\mathrm{t}, J=7.2 \mathrm{~Hz}, 3 \mathrm{H}), 2.54-2.60(\mathrm{~m}, 2 \mathrm{H}), 2.85-$ $2.90(\mathrm{~m}, 2 \mathrm{H}), 3.65(\mathrm{~s}, 3 \mathrm{H}), 4.28(\mathrm{q}, J=7.2 \mathrm{~Hz}, 2 \mathrm{H}), 7.31-$ $7.43(\mathrm{~m}, 5 \mathrm{H}), 7.73(\mathrm{~s}, 1 \mathrm{H})$.

Compound 4: $:^{2 \mathrm{k}} 13 \%$; colorless oil; ${ }^{1} \mathrm{H}$ NMR $\left(\mathrm{CDCl}_{3}, 300\right.$ $\mathrm{MHz}) \delta 2.34(\mathrm{~s}, 6 \mathrm{H}), 3.22(\mathrm{~s}, 2 \mathrm{H}), 7.10(\mathrm{~s}, 1 \mathrm{H}), 7.27-7.45$ $(\mathrm{m}, 3 \mathrm{H}), 7.77-7.81(\mathrm{~m}, 2 \mathrm{H})$.

Acknowledgments. This work was supported by the Korea Research Foundation Grant funded by the Korean Government (MOEHRD, KRF-2006-311-C00384). Spectroscopic data was obtained from the Korea Basic Science Institute, Gwangju branch.

\section{References and Notes}

1. For the Johnson-Claisen tearrangement of Baylis-Hillman adducts, see: (a) Basavaiah, D.; Pandiaraju, S.; Krishnamacharyulu, M. Symlet 1996, 747-748. (b) Das, B.; Majhi, A.; Reddy, K. R.; Venkateswarlu, K. J. Mol. Catal. A: Chem. 2007, 263, 273-275. (c) Das, B.; Majhi, A.; Banerjee, J. Tetrahedron Lett, 2006, 47, 7619-7623. (d) Chapuis, C.; Buchi, G. H.; Wuest, H. Helv. Chim. Acta 2005, 88, 3069-3088. (e) Basavaiah, D.; Pandiaraju, S. Tetrahedron Lett. 1995, 36, 757-758.

2. (a) Lee, H. J.; Kim, H. S.; Kim, J. N. Tetrahedron Lett, 1999, 40, 4363-4366. (b) Lee, H. J.; Chung, Y. M.; Lee, K. Y.; Kim, J. N. Bull. Korean Chem. Soc. 2000, 21,843-844 and the synthesis of compound $\mathbf{2 b}$ was described therein.

3. For the reviews and some recent papers on Eschenmoser-Claisen rearrangements, see: (a) Martin Castro, A. M. Chem. Rev. 2004. 104, 2939-3002, (b) Gilbert, M. W.; Galkina, A.; Mulzer, J. Synlett 2004, 2558-2562. (c) Qu, H.; Gu, X.; Min, B. J.; Liu, Z.; Hruby, V. J. Ong. Lett. 2006, 8, 4215-4218. (d) Schepens, W; Haver, D. V.; Vandewalle, M.; Bouillon, R.; Verstuyf, A.; De Clercq. P. J. Org. Lett. 2006. 8. 4247-4250. (e) Loh, T.-P.; Hu, Q.Y. Org. Lett. 2001, 3, 279-281. (f) Gradl, S. N.; Kennedy-Smoth, J. J.; Kim, J.; Trauner, D. Synlett 2002, $4 \mathrm{Il}-4 \mathrm{l} 4$. (g) Kundig, E. P.; Laxmisha, M. S.; Cannas, R.; Tcherthian, S.; Ronggang, L. Helv. Chim. Acta 2005, 88, 1063-1072. (h) Hill, R. K.; Soman, R, Sawada, S. J. Org. Chem. 1972, 37, 3737-3740. (i) Chen, C.-Y.; Hart, D. J. J. Org. Chem. 1993, 58, 3840-3849. (j) Kundig. E. P.; Cannas, R.; Laxmisha, M.; Ronggang. L.; Tchertchian, S. J. An. Chem. Soc. 2003, 125, 5642-5643.

4. For the synthesis of starting materials including rearranged alcolols and Baylis-Hillman adducts of cyclohexen-2-one, see: (a) Lee, K. Y.; Gowrisankar, S.; Kim, J. N. Bull. Korean Chem. Soc. 2004, 25, 413-414. (b) Lee, K. Y.; Gong. J. H.; Kim, J. N. Bull. Korean Chem. Soc. 2002, 23,659-660.

5. For our recent papers on Baylis-Hillman adducts, see: (a) Lee, K. Y.; Lee, H. S.; Kim, J. N. Tetrahedron Lett, 2007, 48, 2007-2011. (b) Lee, K. Y.; Lee, Y. J.; Kim, J. N. Bull. Korean Chem. Soc. 2007, 28, 143-146. (c) Gowrisankar, S.; Lee, H. S.; Kim, J. N. Bttl. Korean Chem. Soc. 2006, 27, 2097-2100. (d) Lee, K. Y.; Park, D. Y.; Kim, J. N. Bull. Korean Chent. Sac. 2006, 27, 1489-1492. 